\title{
The Effects of Relay Behavior and Position in Wireless Industrial Networks
}

\author{
Svetlana Girs \\ Mälardalen University \\ Västerås, Sweden \\ svetlana.girs@mdh.se
}

\author{
Elisabeth Uhlemann \\ Halmstad University \\ Halmstad, Sweden \\ elisabeth.uhlemann@ieee.org
}

\author{
Mats Björkman \\ Mälardalen University \\ Västerås, Sweden \\ mats.bjorkman@mdh.se
}

\begin{abstract}
In wireless industrial networks, providing reliable and timely communication is crucial. Previous research has shown that relaying can be used to achieve this goal. However, we have found that different types of wireless channels encountered in industrial environments affect the benefits as well as the best behavior and position of relay nodes. We consider two types of behavior: the relay node always retransmits or it only retransmits if it has obtained a correct copy of the packet. We evaluate different positions of the relay node by conducting simulations for two types of fading channels: with and without line of sight. The results clearly show that the benefits, the best behavior and position of a relay node depends on the wireless channel, whether or not the bit errors appear randomly or in bursts and also on the distance between the source and the final destination.
\end{abstract}

\section{Introduction}

The main difference between wireless industrial networks and other wireless networks is that the former have to satisfy strict requirements in terms of real-time deadlines and high reliability at the same time, for example when an important alarm signal is transmitted. It is well known that wireless channels introduce bit errors, as results of interference, noise, path loss, shadowing or fading. All these factors imply a significant challenge to fulfill the real-time and reliability requirements.

A great variety of error control schemes exist that can tackle the effects of the wireless channel. These can be broadly divided into forward error-control coding (FEC) and retransmission-based automatic-repeat-request (ARQ) schemes. FEC is the best choice in situations where no feedback channel for ARQ is present, but also in communication environments where the number of errors is moderate, e.g., wired fieldbuses, such that the FEC is able to correct the errors. However, for very challenging wireless environments, where errors appear in bursts that are longer than the error correction capability of the code, FEC is less useful. ARQ, on the other hand, works best when errors appear in burst as the benefits of time-diversity then comes into play, i.e., when the re-

E. Uhlemann is partly funded by the Swedish Governmental Agency for Innovation Systems, Vinnova, through the VINNMER program, www.vinnova.se transmission takes place the instantaneous channel situation is likely to have changed. However, if errors do not appear in bursts, one single bit error can cause a whole packet of several bytes to be retransmitted. In this case ARQ may lead to excessive retransmissions such that deadlines are missed. A combination of the two, called Hybrid ARQ can then be used. Hybrid ARQ combines the benefits of FEC and ARQ such that situation with few errors are handled by the FEC code, whereas a burst of errors leads to a retransmission and thus a new attempt for the FEC code. For industrial communications, where packets have real-time constraints, the number of allowed retransmission is limited by the deadline. It is then important to find a FEC code that is powerful enough. In the last years, FEC techniques such as turbo codes and iterative decoding algorithms were developed that can operate closely to information-theoretical limits. Unfortunately the usefulness of these codes for industrial applications is limited because of the fact that they can play out their advantages only for large packet sizes, while most industrial applications use rather short packets.

A fundamental approach to increase reliability of industrial wireless networks using short packets is the exploitation of spatial diversity [1]. In this class of mechanisms, the single-channel restriction between the source and the destination is removed and information is transmitted over multiple spatial channels. These channels are stochastically independent and the probability of them being in a deep fade at the same time is very small. There are several spatial diversity techniques which can be used to increase network reliability: multiple-input multiple-output (MIMO) schemes, relaying or cooperative communication, network coding, distributed spacetime coding, cooperative coding or fountain codes. In industrial networks, devises are limited by size and hardware complexity to one antenna, [2], and therefore MIMO techniques can typically not be used. Also, as it is usually required to transmit data from one sensor to one central control node, we cannot benefit from using network coding, which gives best results in many-tomany broadcast networks, or fountain codes which usually are used for one-to-many communications. In addition, the use of low complexity, off-the-shelf components in industry generally prevents the use of sophisticated space-time codes. However, the idea of cooperative diversity using relaying which can be implemented on top of off-the-shelf transceivers is promising $[1,3]$. 
The concept of relaying is not new, and the first theoretical work was done in seventies [4]. One of the first examples of practical cooperative diversity protocols was proposed by Laneman, Tse and Wornell in [5]. In relaying schemes, e.g., [6], there are a number of relay nodes that help in the transmission between a source and a destination node. These relay nodes may receive the packet correctly, even if the destination does not and then they can retransmit it to the final destination. The key advantage is that due to the fact that the source and the relay nodes have different geographical locations, it is possible to exploit spatial diversity. In the last years, a great deal of information-theoretical research as well as research on practical integration of relaying has been carried out. While implementing a relaying protocol into wireless industrial networks, some significant questions should be answered [7]. First of all it is important to decide if relaying is desired (i.e. if there is time left before the deadline to do a relaying attempt) and required (it is not required in case of successful direct transmission). Given that relaying is desired and required, the third question is how to choose the position of the relay node and its behavior algorithm. A simple and practically implementable protocol in which relaying and packet combining work together to improve reliability in deadline constrained networks was designed in [3]. However, the choice of position and behavior for the relay nodes were only examined for one type of channel, a fading channel without line of sight. Some research on best relay node placement was done in, e.g., [8, 9]. In [8] the authors present a wireless relay placement algorithm for wireless sensor networks, which attempts to reduce the sensoraverage probability of error. In the case of industrial communications, the worst case error probability is of essence as we consider real-time data traffic. Further [8] assumes no retransmission scheme, and instead each packet is always transmitted twice, from the source and from the relayer, and a diversity combining algorithm is made at the final destination. The results from [9] shows that the choice of path loss model and fading distribution model effect the optimal amount of relay nodes as well as their positions. This conclusion is similar to ours, but the algorithms from [9] cannot be directly applied as it considers only one specific kind of environment - underground tunnels.

In this paper for a given source and destination as well as a given wireless channel, we want to find the best position of the relay node together with the most suitable acting strategy for it. We consider two types of behavior: the relay node always retransmits whenever a retransmission is requested or it only retransmits if it has obtained a correct copy of the packet - otherwise the source handles the retransmission. To answer the question stated above we evaluate the protocol performance for two types of channels: Rician and Rayleigh fading channels representing with and without line of sight respectively.

The reminder of this paper is structured as follows: in Section II different wireless channels encountered in in- dustrial environments are briefly discussed. Phenomena such as fading, multipath propagation, shadowing and path loss are treated. Next, in Section III system model is described. Following this, in Section IV we describe the relaying schemes used in this paper and in Section IV we provide simulation results comparing these schemes for different placements of the source, the relay and the destination nodes. We conclude the paper in Section V.

\section{Wireless industrial environments}

Wireless industrial channels are very challenging environments as they contain disturbances from electromechanical machinery and high reflective facilities, which makes reliable and timely communication more complicated. Several different factors affect the received signal: pathloss, thermal noise, multipath interference and shadowing. The pathloss is a loss in signal strength depending on the distance between the source and the destination. Of course, a signal that propagates in vacuum or water has a different pathloss than one that travels though air, but in general it is a deterministic value. Thermal noise is present in all electronic devices and is caused by electron movement. Although the probability density function (pdf) for electron movement is not known, thermal noise can be modeled as additive white Gaussian noise (AWGN), due to the law of large numbers. For certain space communication channels, AWGN is sufficient to model the channel as no objects are nearby and the pathloss is negligible in space. However, in most other wireless channels different objects, like the ground, are located close to, or even in-between the source and the destination. Waveforms encountering these objects can be subject to reflections, scattering and diffraction, and as a result, multiple copies of the same packet can reach the receiver. Since the copies have traveled through different paths to get to the receiver, they may arrive with different delay and different attenuation. Interference between these multiple copies can therefore be constructive or destructive and the signal is said to experience fading. In case of destructive interference the channel is often said to be in a deep fade. Such deep fades also occur due to shadowing. Since in a wireless channel things can move in and out of the line-of sight between the transmitter and the receiver, shadowing can occur suddenly and remain for a short or long duration.

In this paper we use AWGN to model the thermal noise, and a log-normal distribution to model the pathloss. In addition, two types of fading channels are examined: Rician and Rayleigh fading channels. During Rician fading, the signal travels through a line-of-sight (LOS) path as well as multiple reflective non-LOS paths. The $K$-factor of the Rician channel determines how strong the LOS path is compared to the other paths. For $K=0$, which is the same as Rayleigh fading, there is no LOS at all, and all communication takes place using multiple non-LOS paths. In contrast, then the $K$ factor goes to infinity, the channel becomes AWGN, i.e., only one strong LOS path. Since wireless industrial channels 
contain much machinery and reflective metallic facilities, AWGN is not a good model even if there is a clear LOS present. Therefore we consider the Rician channel with different $K$ factors, to model the presence of a LOS path, together with different amounts of machinery causing multipath fading. Our results for the Rayleigh channel can be applied in case of shadowing, i.e. when something moves into the LOS between the source and destination. A lot of work has been done to characterize industrial communication environments. In [10] the authors carried out a set of measurements to evaluate the impact of industrial disturbances on wireless communication. Some work on determining the best approximation for wireless industrial channels has been done before, e.g., in [11] the authors concluded that the Rician distribution with $K$-factor 20 is the best-fit fading model for short range wireless sensor networks. On the other hand, the authors of [12], after carrying out a set of measurements at various industrial locations, concluded that it is unclear if a single distribution can be used to characterize the channel. Most of their results exhibited Nakagami- $m$ characteristics, while some of them exhibited Lognormal and Rayleigh characteristics. We have thus used $K$-factor 20 and 5 as well as the Rayleigh channel to model multipath propagation.

\section{System model}

In this section we describe the network setup, the channel model and the performance measure considered in this paper.

\subsection{Network setup}

We consider the network with three nodes: a source, a relay and a destination node. All the nodes are halfduplex transceivers, working on the same frequency and with the same data transmission rate. The distance between the source and the destination is $d$. In scenarios with a relay node, it is placed in between the source and the destination. For different simulations we used different positions: $0.25 d, 0.5 d$ and $0.75 d$, Fig. 1 .

The value $0.25 d$ refers to a situation where the distance between source and relay is $25 \%$ of $d$ and thus the distance between relay and destination is $75 \%$ of $d$.

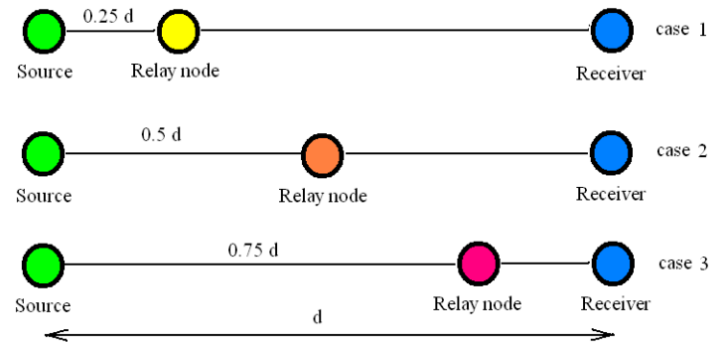

Fig. 1. Investigated node deployment

Two assumptions were adopted in our protocol. The first assumption is about the way the feedback information is delivered. We assume that the source and the relay node know if a retransmission is needed and that the source knows if the relay node is going to retransmit or not. Thus our results can be considered as the lower bound for the possible packet error rate. We adopt this assumption as it helps us to evaluate in general how relaying affects the resulting packet error rate. If relaying turns out to be beneficial, our future work will be to find a scheme to provide efficient feedback information, knowing about the lower bound.

The second important assumption in our protocol is that relay node can work in promiscuous mode, where the hardware allows to switch off automated CRC checking. We use this mode in "always relay" scheme, where a relay node can accept (possible erroneous) packets from its transceiver and forward them.

\subsection{Channel model}

For each pair of nodes there is a wireless channel with behavior which is stochastically independent from all other channels. We assume that all channels are symmetric and all nodes are stationary. The additional interference from other systems is not taken in to account. Thermal noise created in the receiver circuitry, $n(t)$, is also a parameter in our model and assumed to be AWGN with power spectral density $N_{0} / 2$. The log-distance model [13], $l(d)$, is adopted in this paper as a path loss approximation, implying that the signal strength decreases proportionally to $d^{\prime \prime}$, where the pathloss component, $\gamma$, is chosen as $\gamma=2$. We also assume frequency-flat block Rayleigh or Rician fading, $h(t)$, on each pathloss AWGN channel. It is considered that the fading remains constant during the whole period of one packet transmission. The block-fading assumption is justified by the dominance of small packets in industrial applications. For our simulations we choose a packet size of 160 bits. We draw the value of $h(t)$ periodically from Rayleigh or Rician distribution and keep it constant for the duration of the packet. The resulting received signal can be expressed as $r(t)=l(d) h(t) s(t)+n(t)$, where $s(t)$ is the transmitted signal. We assume binary phase shift keying (BPSK) modulation.

The signal-to-noise ratio ( $\mathrm{SNR}), E_{s} / N_{0}$, where $E_{s}$ is the received signal energy per transmitted bit and $N_{0}$ is the noise power spectral density, changes with the distance between the source and the destination, $d$. We have evaluated different values of $E_{s} / N_{0}$ and then we use the settings from the standard of IEEE 802.15.4 to transform $E_{s} / N_{0}$ into $d$. This implies that $E_{s} / N_{0}=P_{t} l(d) /\left(R N_{0}\right)$, where $P_{t}$ is the transmitted signal power $(1 \mathrm{~mW}$ in 802.15.4), $l(d)$ is the pathloss, i.e., $l(d)=l\left(d_{0}\right) 10 \gamma \log$ $\left(d_{0} / d\right)$, where $l\left(d_{0}\right)=-50 \mathrm{~dB}$ at the reference distance $d_{0}$ $=1 \mathrm{~m}$ usually is assumed, $R$ is the transfer rate $(250$ kbps in 802.15.4) and $N_{0}$ is the thermal noise in the receiver circuitry $(-173 \mathrm{dbW} / \mathrm{Hz}$ in 802.15 .4$)$.

\subsection{Performance measure}

The performance measure adopted in this paper is the success ratio, i.e., the per cent of packets that are correctly received before their deadline as a function of the distance between the source and the final destination, $d$. 
We assume that one retransmission or relaying attempt is allowed before the deadline and thus the success ratio is equal to one minus the resulting packet error rate (PER) including a retransmission when required. For our numerical evaluations, we also consider the PER as a function of $d$.

\section{Protocol design}

In this section we explain how the relaying schemes are organized, how the relay node determines if it should retransmit the packet or if it is better to let the source retransmit. Also we introduce theoretical expressions, which we use to verify the correctness of the results from our simulations. We evaluated three different scenarios: "ARQ but no relay", "always relay" and "only relay when correct". We call the last two strategies "always relay" and "only relay when correct", since they cannot be directly translated to the commonly used nomenclature "decode-and-forward" and "amplify-and-forward" as we only have access to the decoded bits and not the received observables, due to the use of commercial transceivers.

\subsection{ARQ but no relay}

According to this scheme, one retransmission from the source node is allowed, if the destination node detects any bit errors in the received packet. For this case the PER is

$$
P_{\text {noRelay }}(d)=P_{\text {noARQ }}(d)^{2}
$$

where

$$
P_{\text {noARQ }}(d, L) \approx 1-\left(1-P_{b}(d)\right)^{L},
$$

and $P_{b}$ is the probability of bit error for a system without retransmissions, $d$ is the distance between the source and the final destination and $L$ is the length of the packet. Note that closed form expressions for $P_{b}$ depending on $d$ and the channel type, Rician or Rayleigh, can be found in e.g., [14]. The only time the equality holds in (2) is when all bit errors are uniformly distributed between packets. When errors appear in bursts, (2) is an approximation. For simplicity we will drop the packet length, $L$, since all transmissions use packets of the same length. We assume that this is the case we should benchmark our relaying schemes against, namely, a system where one and only one retransmission is allowed due to timing constraints dictated by the deadline.

\subsection{Always relay}

In this scenario we allow one relaying attempt. While working according to the "always relay" algorithm, the relay node retransmits the message without checking if it is correct of not. The nodes work as follows: the source node transmits the original packet; the relayer and the destination listen to receive it. The destination node checks the correctness of the packet. If it is not correct, the relay node retransmits the packet, i.e., the relay node sends the packet even if the CRC check has failed. For this case packet errors can occur if the direct transmission between the source and the destination failed and then either there were errors in the packet received from the source node by the relay node, or if the relaying attempt was unsuccessful, i.e., errors in the packet received by the destination from the relay node. The PER is

$$
\begin{aligned}
& P_{\text {always }}(d)=P_{\text {noARQ }}(d) P_{\text {noARQ }}\left(d_{S \rightarrow R}\right)+ \\
& P_{\text {noARQ }}(d)\left(1-P_{\text {noARQ }}\left(d_{S \rightarrow R}\right)\right) P_{\text {noARQ }}\left(d_{R \rightarrow D}\right),
\end{aligned}
$$

where $d_{S \rightarrow R}$ and $d_{R \rightarrow D}$ are the distances between the source and relay node and the relay and destination node respectively. In (3) we have assumed that if a bit error occurs on the transmission from the source to the relayer, it is not corrected during the transmission from the relayer to the final destination.

\subsection{Only relay when correct}

The "only relay when correct" scheme assumes that the relay node checks the packet first and sends it only if it has a correct copy. As always, the source first transmits the original packet, the relay and the destination node listen to try to receive it and check the CRC correctness. If the received packet contain errors, the relayer can retransmit the packet but only if it has a correct copy. If the packet is erroneous at the relay node, it does not transmit anything. Instead, the source node retransmits the packet. For this scheme errors can occur if the direct transmission was unsuccessful and there were errors in the packet, received by relay node, followed by errors in retransmission from the source, or if the relaying attempt failed. The probability of packet error is given by

$$
\begin{aligned}
& P_{\text {onlycorrect }}(d)=P_{\text {no ARQ }}(d)^{2} P_{\text {noARQ }}\left(d_{S \rightarrow R}\right)+ \\
& P_{\text {no ARQ }}(d)\left(1-P_{\text {noARQ }}\left(d_{S \rightarrow R}\right)\right) P_{\text {noARQ }}\left(d_{R \rightarrow D}\right) .
\end{aligned}
$$

\section{Results}

In this section we present the results from comparison of our relaying ARQ schemes with different parameters. We looked at two types of figures: success ratio, i.e. the per cent of the correctly received packets before the deadline as a function of distance between the source and the final destination, $d$, and PER versus relay node location for fixed values of $d$. For reference, one ARQ scheme without relaying is also plotted on all of the pictures: the scheme allowing one retransmission but from the source only, i.e., no relaying.

\subsection{Rayleigh fading channel}

First we consider the case of a channel with Rayleigh fading, i.e., no LOS. Fig. 2 shows the success ratio while a relay node, working according to the "only relay when correct" algorithm, is placed in three different positions. 
The relayer is placed at $d / 4, d / 2$, or $3 d / 4$, where $d$ is the distance between the source and the destination. In the legend "relay at $25 \%(50 \%$ or $75 \%)$ " means that the relay node is situated at the distance $0.25 d(0.5 d$ or $0.25 d)$ from the source and $0.75 d(0.5 d$ or $0.25 d)$ from the destination node.

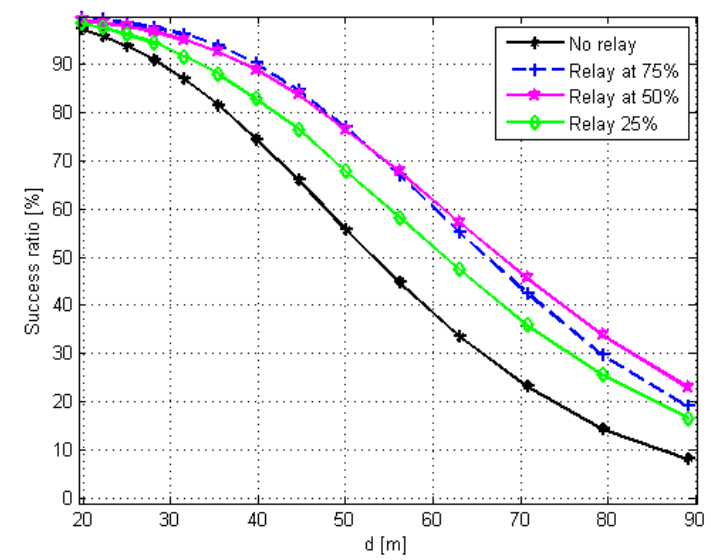

Fig. 2. The success ratio for schemes with relay nodes at different locations (Rayleigh channel, "only relay when correct")

It can be clearly seen from the figure that usage of a relay node at any of the three positions leads to performance benefits in comparison with a scheme without relaying. The best position of the relay node depends on the overall distance between the source and destination. In particular, for large $d$, it is best to place the relay node at $50 \%$ of the overall distance between the source and the destination. However, when $d$ is below $50 \mathrm{~m}$, it is better to place the relay node closer to the destination. Also note that from (4) it can be seen that having a relay at $25 \%$ is not the same as having one at $75 \%$ even though two consecutive transmissions over the same total distance is made, which explains why a relay node at $25 \%$ of $d$ is never the best option.

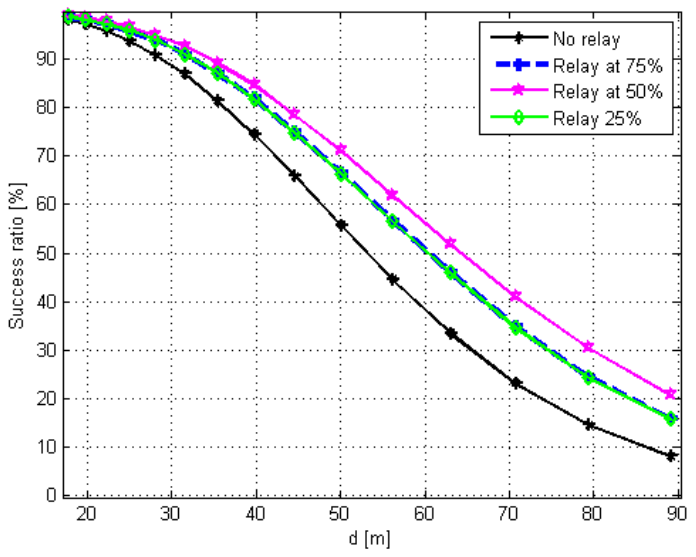

Fig. 3. The success ratio for schemes with relay nodes at different locations (Rayleigh channel, "always relay")

In Fig. 3 the success ratio for different $d$ is shown when the relay node works according to the "always re- lay" scheme. In this case having a relayer, located exactly in the middle between the source and the destinations gives the highest amount of correct packets for any $d$.

Fig. 4 lets us compare the results of the two different relay node behavior scenarios: "always relay" and "only relay when correct". The relay node is located at $0.5 d$ or at $0.75 d$ from the source. We chose these locations as they showed the highest success ratio, according to findings in Fig. 2 and Fig. 3.

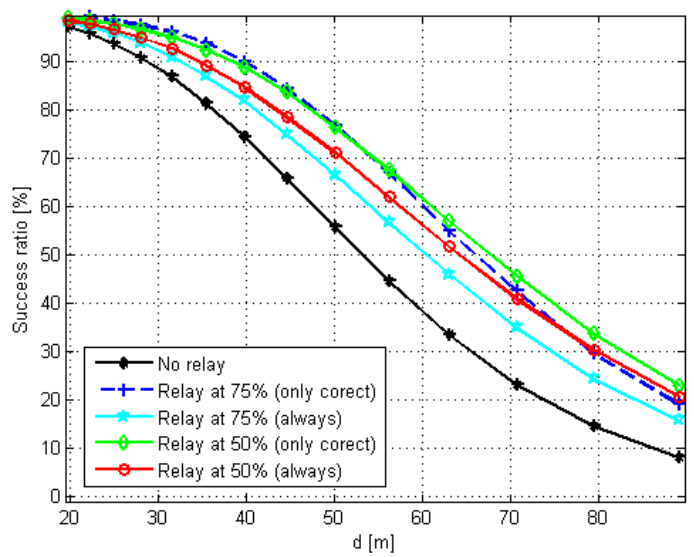

Fig. 4. The effect of a relay node, using two different relay strategies (Rayleigh channel)

It is seen from the figure, that for both positions of the relay node, the highest success ratio can be achieved while using the "only relay when correct" scenario. This result is the effect of the type of signal strength variation in the used channel. In a channel with severe fading, such as in Fig. 4, errors appear in bursts, i.e. the major part of a short packet can be corrupted. That means that if the relay node gets an erroneous copy of the packet, it is better to let the source retransmit rather than relaying the corrupted packet.

\subsection{Rician channel}

Next we show the simulation results for Rician channel with $K$-factor 5 and 20. A $K$-factor $=5$ represents the situation where there is a LOS component, but it is not very strong. The figure for $K$-factor 20 , corresponding to a good, strong LOS path, is presented here both because it is interesting to see if it makes a difference to place relayers with the aim of obtaining a good strong line of sight and because in some evaluations, e.g., [11], it was found to be the best-fit value for industrial conditions.

Fig. 5 and Fig. 6 show the Rician channel with $K$ factor $=5$, while using three different relay node positions. The relay node works according to the "only relay when correct" algorithm in Fig. 5, and the "always relay" in Fig. 6. We can conclude that the best position of the relay node for both cases is $0.5 d$. For distances smaller than $30 \mathrm{~m}$ the difference between the two positions 0.5 and 0.75 diminishes. 


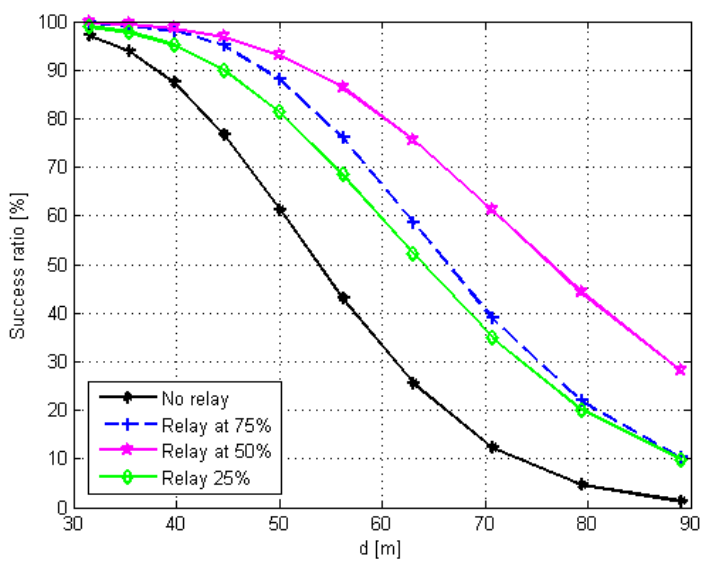

Fig. 5. The success ratio for schemes with relay nodes at different locations (Rician channel, $K$-factor $=5$, "only relay when correct")

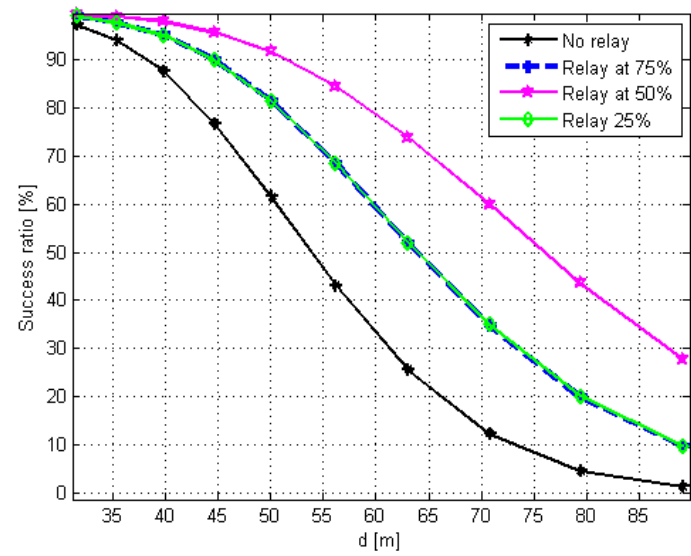

Fig. 6. The success ratio for schemes with relay nodes at different locations (Rician channel, $K$-factor $=5$, "always relay")

The next group of figures show the results for a Rician channel with $K$-factor $=20$. For this channel, as it can be seen from Fig. 7 and Fig. 8, the best performance can be achieved using a relay node at $50 \%$, especially for large distances between the source and the destination.

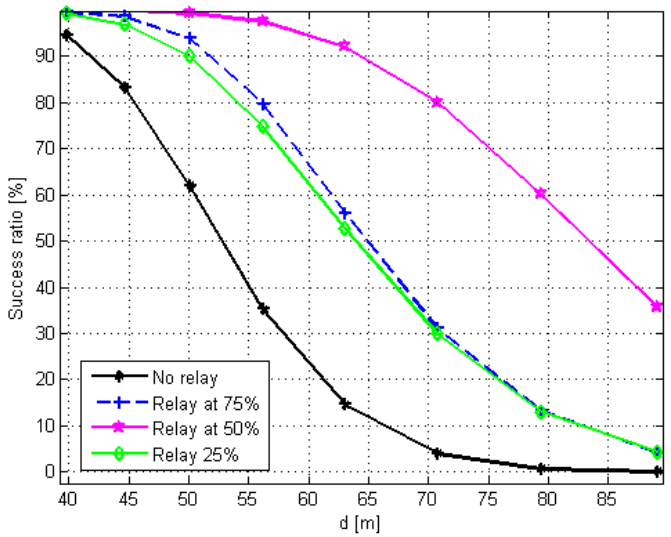

Fig. 7. The success ratio for schemes with relay nodes at different locations (Rician channel, $K$-factor $=20$, "only relay when correct")

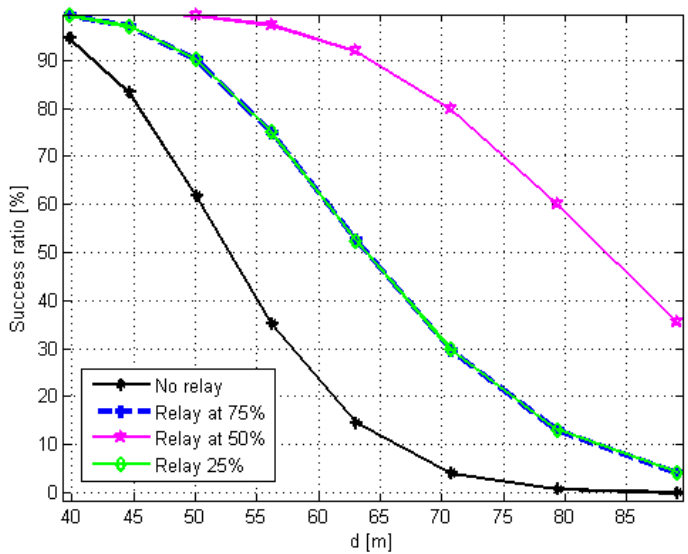

Fig. 8. The success ratio for schemes with relay nodes at different locations (Rician channel, $K$-factor $=20$, "always relay")

In Fig. 9 the benefits of using relaying for Rician channels with $K$-factor $=5$ and 20 are compared when the relay node uses the algorithm "only relay when correct". The results show that for larger distances, over 45 $\mathrm{m}$, the channel with $K$-factor $=20$ and the relay node at $50 \%$ achieves the best performance. For shorter communication ranges, the difference in success ratio between the two schemes is not so big.

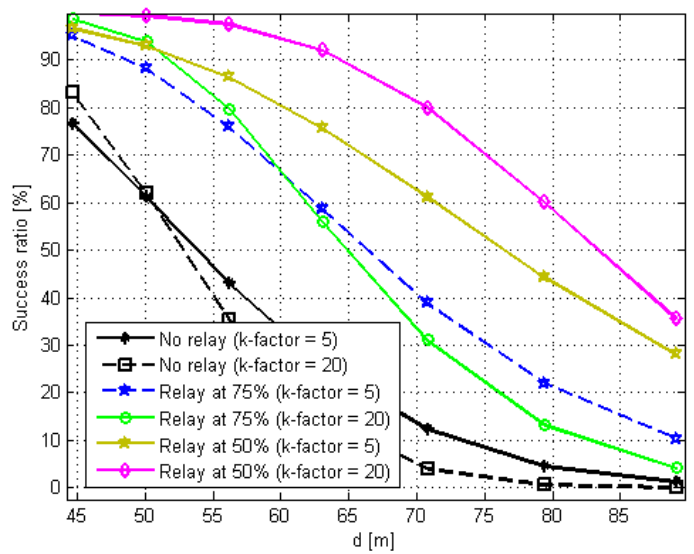

Fig. 9. Comparison between the results for Rician channel with $K$-factor $=5$ and 20 ("only relay when correct")

\subsection{Theoretical PER}

In our simulations, it is difficult to evaluate the success ratio for small distances, i.e., smaller than $50 \mathrm{~m}$. This is due to the fact that the bit error probability is around $10^{-5}$ already for these distances and the random number generator in the simulation program is not accurate enough to evaluate these low numbers by MonteCarlo simulations. However, using our theoretical expressions in Section 4, we are able to evaluate distances smaller than $50 \mathrm{~m}$. Note, however, that there is an approximation in our theoretical evaluation, i.e., we assume that bit errors are uniformly distributed among packets. However, the numerical evaluations should still be more accurate than the simulations for short dis- 
tances. Fig. 10 and Fig. 11, showing theoretical PER versus the position of the relay node, were thus obtained from the mathematical expressions given in Section 4. In each figure, there are curves for all three examined channels: $K$-factor $=0$ means a Rayleigh channel without LOS, whereas $K$-factors $=5$ and 20 correspond to a $\mathrm{Ri}$ cian channel. The value $10 \%$ on the $\mathrm{x}$-axis means that the relayer is very close to the source, while the $100 \%$ position is equal to a dual receiving antenna.

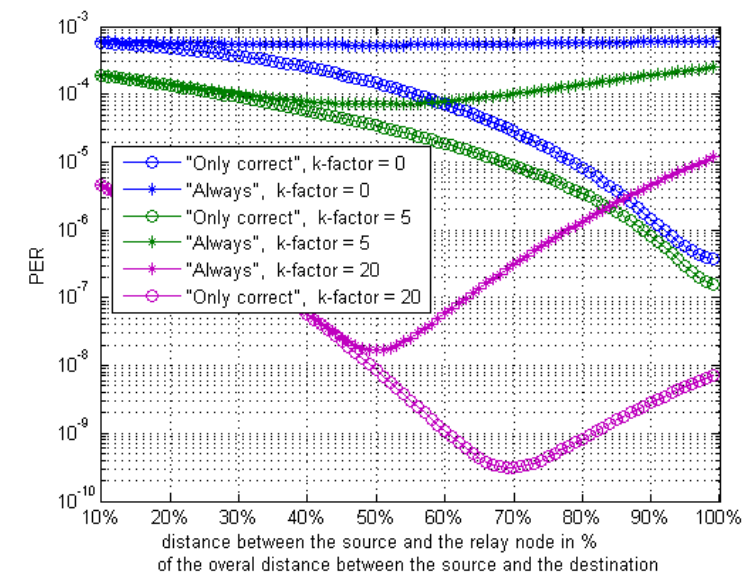

Fig. 10. PER versus the distance between the source and the relay node for all three channels $(d=28[\mathrm{~m}])$

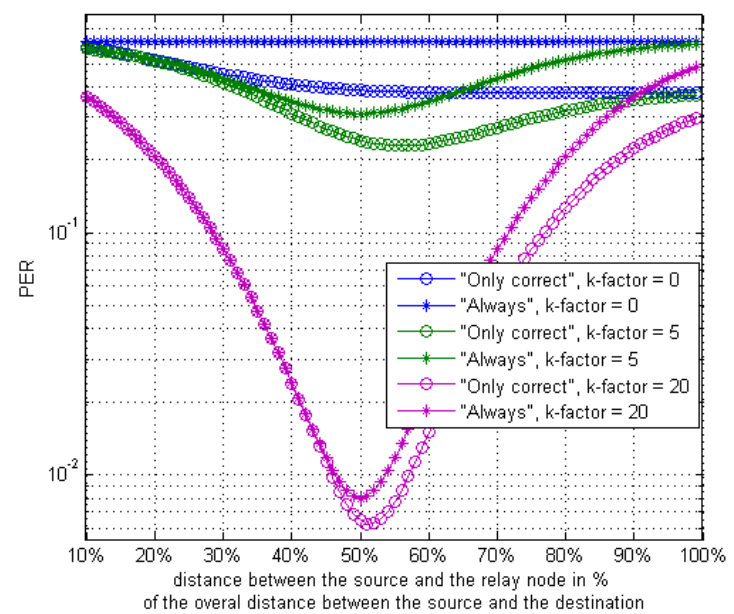

Fig. 11. PER versus the distance between the source and the relay node for all three channels $(d=50[\mathrm{~m}])$

It can be seen from the figures that for all three channels, and both $d$ values, the best behavior of the relay node is to "only relay when correct". Further, we can see that if the relay node is working according to the "always relay" algorithm, the best position of the relay node is $50 \%$. For the "only relay when correct" case, the choice of the position of the relayer depends on the channel (how strong the LOS is) and the overall distance between the source and the destination. For a channel without LOS, the best location of the relay node for smaller distances is as close as possible to the destina- tion, which means that we approach multiple receiving antennas. For larger distances, the minimum PER is obtained somewhere in-between $50 \%$ and $100 \%$, depending on $d$ (it cannot be seen directly from Fig. 11, but for $d=50$, there is a minimum PER at 78\%). When a LOS path is present at smaller distances, the best choice depends on the strength of the LOS path. For a weak LOS path, positions closer to the destination gives better results, whereas when the LOS path becomes more dominating, a relay position around $69 \%$ is best. These conclusions are further substantiated when considering larger distances between the source and the destination: for a relatively weak LOS path placing the relay node at around $57 \%$ is best, whereas for strong LOS channels, the best option is to place the relay node midway inbetween the source and destination.

It should also be noted that if errors are few and their distribution is close to uniform due to a strong LOS, at the same time as the overall distance between the source and the final destination is moderate, the choice of relay behavior is not so obvious. Letting the relay node always retransmit results in a lower bit error rate, but still gives the same or in some cases a bit worse PER than if it only retransmits when it has a correct copy of the packet. This cannot be seen from Fig. 11 as it only shows PER, but given a good FEC code and packet combining, which benefits from a low bit error rate, it is better to use the scheme "always relay".

\section{Conclusions}

The main goal of this paper was to evaluate how different types of communication channels encountered in wireless industrial environments affect the choice of relaying scheme. We built several simulation models and tried different relaying scenarios for two types of fading channels. From the simulation results it can be clearly seen that the benefit of using relaying is very different depending on the type of channel. If bit errors appear in bursts, i.e., the LOS path is absent or weak compared to the non-LOS paths, it is better to only allow a relay node to perform a retransmission if it has obtained a correct copy of the packet in question. However, if errors are few and close to uniformly distributed due to a strong LOS, at the same time as the overall distance between the source and the final destination is moderate, the choice of relay behavior is not so obvious. Allowing the relay node to handle all retransmissions results in a lower bit error rate, but still gives the same or in some cases a bit worse PER than only letting the relay node handle retransmissions when it has a correct copy of the packet. However, for some industrial applications no packet errors can be tolerated and in this case the "always relay" strategy should only be used in conjunction with a good FEC or packet combining.

Our results also show that careful placement of relay nodes such that a good LOS path is obtained, improves performance significantly. If a strong LOS is present, it is best to place the relay node close to midway (50-70\%) 
between the source and the destination, such that it is used as a multihop node. However, for a weak LOS path, or even no LOS, it is better to place the relayer very close to the destination, such that it can act as a virtual dual antenna to combat the fading characteristics The placement of the relay node is not only dependent on LOS, but also on the relative distance between the source and the final destination. If the source and the destination are well within range of each other, positions closer to the destination achieves the best results, whereas when the source is located far away from the destination, positions closer to $50 \%$ is better.

Thus, as the next step we plan to carry out a set of measurements on the territory of real industrial settings. From the measured data we plan to build a set of channel models which can be used for industrial environment characterization as well as for evaluating our relaying schemes.

\section{References}

[1] S. N. Diggavi, N. Al-Dhahir, A. Stamoulis, and A. R. Calderbank, "Great Expectations: The Value of Spatial Diversity in Wireless Networks", Proceedings of the IEEE, vol. 92, no. 2, pp. 219-270, February 2004.

[2] A. Willig, K. Matheus, and A. Wolisz, "Wireless Technology in Industrial Networks," Proceedings of the IEEE, vol. 93, no. 6, pp. 1130-1151, June 2005.

[3] E. Uhlemann and A. Willig, "Joint design of relay and packet combining schemes for wireless industrial networks", in Proc. IEEE Vehicular Technology Conference, Singapore, May 2008, pp. $2441-2445$.

[4] T. M. Cover and A. El Gamal, "Capacity Theorems for the Relay Channel", IEEE Transactions on Information Theory, vol. 25, no. 5, pp. 572-584, September 1979.
[5] N. Laneman, D. Tse, and G. Wornell, "Cooperative Diversity in Wireless Networks: Efficient Protocols and Outage Behavior," IEEE Transactions on Information Theory, vol. 50, no. 12, pp. 3062-3080, December 2004.

[6] A. Nosratinia, T. E. Hunter, and A. Hedayat, "Cooperative Communication in Wireless Networks," IEEE Communications Magazine, vol. 42, no. 10, pp. 74-80, October 2004.

[7] A. Willig, "How to Exploit Spatial Diversity in Wireless Industrial Networks," in IFAC Annual Reviews in Control, vol. 32, no. 1, pp. 49-57, April 2008.

[8] J. Cannons, L. B. Milstein, K. Zeger, "An Algorithm for Wireless Relay Placement", IEEE Transactions on Wireless Communications, vol. 8, no. 11, pp. 5564 5574, November 2009.

[9] R. Liu, I. J. Wassell, K. Soga, "Relay node placement for wireless sensor networks deployed in tunnels", in Proc. IEEE International Conference on Wireless and Mobile Computing, Networking and Communications, Niagara Falls, ON, October 2010, pp. $144-150$.

[10] C. Angskog, C. F. Coll, C. Chilo and P. Stenumgaard, "Sources of disturbances on wireless communication in industrial and factory environments", in Proc. AsiaPacific Symposium on Electromagnetic Compatibility, Bejing, China, April 2010, pp. 281-284.

[11] F. Darbari, I. A. Glover and R. W. Stewart, "Channel and interference analysis for wireless sensor networks," in Proc. IEEE International Conference on Communications, Glasgow, UK, June 2007, pp. 3289-3294.

[12] D. Sexton, M. Mahony, M. Lapinski and J. Werb, "Radio channel quality in industrial wireless sensor networks," in Proc. Sensors for Industry Conference, Houston, Texas, February 2005, pp. 88-94.

[13] T. S. Rappaport, Wireless Communications - Principles and practice: Prentice-Hall, 1996.

[14] A. Goldsmith, Wireless Communications, Cambridge University Press, New York, 2005. 